\title{
Singular Instantons and Creation of Open Universes
}

\author{
Alexander Vilenkin* \\ Institute of Cosmology, Department of Physics and Astronomy, \\ Tufts University, Medford, Massachusetts 02155, USA
}

(October 24, 2018)

\begin{abstract}
Singular instantons of the type introduced by Hawking and Turok lead to unacceptable physical consequences and cannot, therefore, be used to describe the creation of open universes.
\end{abstract}

*Electronic address: vilenkin@cosmos2.phy.tufts.edu 
In a recent paper [1], Hawking and Turok presented an instanton solution which they interpret as describing the creation of an open inflationary universe. An unusual feature of this instanton is that it is singular : both the curvature and the scalar field diverge at one point. Hawking and Turok argue that it is nonetheless a legitimate instanton, since the singularity is integrable and the Euclidean action is finite. I do not find this argument very convincing. In a singular instanton, the field equations are not satisfied at the singularity. Such an instanton is not, therefore, a stationary point of the Euclidean action, and it is not at all clear that it will give a dominant contribution to the Euclidean path integral. Moreover, if the Hawking-Turok(HT) instanton is allowed, we will then have to admit a host of other instantons with integrable singularities. Here, I shall give an example of such an instanton and argue that it leads to physically unacceptable consequences.

Let us consider the model of a massless scalar field $\phi$ interacting with gravity. The corresponding Euclidean action ist

$$
S_{E}=\int d^{4} x \sqrt{g}\left[-\frac{R}{16 \pi G}+\frac{1}{2}(\partial \phi)^{2}\right]+S_{\text {boundary }} .
$$

As in [1], we shall be interested in an $\mathrm{O}(4)$-symmetric instanton described by the metric

$$
d s^{2}=d \sigma^{2}+b^{2}(\sigma)\left(d \psi^{2}+\sin ^{2} \psi d \Omega_{2}^{2}\right)
$$

with $\phi=\phi(\sigma)$. The field equations for $b(\sigma)$ and $\phi(\sigma)$ are

$$
\begin{gathered}
\phi^{\prime \prime}+3 \frac{b^{\prime}}{b} \phi^{\prime}=0, \\
b^{\prime \prime}=-\frac{8 \pi G}{3} b \phi^{\prime 2},
\end{gathered}
$$

where primes stand for derivatives with respect to $\sigma$. We choose the boundary conditions corresponding to an asymptotically-flat instanton,

$$
b(\sigma) \approx \sigma, \quad \phi(\sigma) \rightarrow 0 \quad(\sigma \rightarrow \infty)
$$

¿From (3) it follows that

$$
\phi^{\prime}=-\frac{C}{b^{3}},
$$

where $C=$ const. Substituting this in (4) and integrating, we have

$$
b^{\prime 2}-\frac{4 \pi G C^{2}}{3 b^{4}}=1
$$

where the constant of integration has been chosen according to the boundary condition (5). The asymptotic forms of the solution at large and small $\sigma$ are easily found. At $\sigma \rightarrow \infty$,

\footnotetext{
${ }^{1} \mathrm{~A}$ massless field has been chosen for simplicity. A similar instanton can easily be constructed with a massive scalar field.
} 


$$
\begin{gathered}
b(\sigma) \approx \sigma+\mathcal{O}\left(\sigma^{-3}\right), \\
\phi(\sigma) \approx C / 2 \sigma^{2} .
\end{gathered}
$$

As we move towards smaller values of $\sigma, b(\sigma)$ decreases and $\phi(\sigma)$ grows until $b$ vanishes and $\phi$ diverges at some $\sigma=\sigma_{*} \sim\left(C / m_{p}\right)^{1 / 2}$. The form of the solution near $\sigma_{*}$ is given by

$$
\begin{gathered}
b(\sigma) \approx\left(12 \pi G C^{2}\right)^{1 / 6}\left(\sigma-\sigma_{*}\right)^{1 / 3}, \\
\phi(\sigma) \approx(12 \pi G)^{-1 / 2} \ln \left(\sigma-\sigma_{*}\right)+\text { const } .
\end{gathered}
$$

The transition between the two regimes occurs at $\sigma \sim\left(C / m_{p}\right)^{1 / 2}, \phi \sim m_{p}$, where $m_{p}$ is the Planck mass. The singular behavior (10), (11) is identical to that of HT instanton. So if HT instanton is legitimate, then we have no reason to reject the asymptotically-flat instanton described above.

To evaluate the Euclidean action of our instanton, we first note that the trace of Euclidean Einstein equations reads $R=8 \pi G(\partial \phi)^{2}$. Hence the two terms in the square brackets of Eq. (1) cancel, and the only contribution to the action comes from the boundary term at $\sigma=\sigma_{*}$. This term was omitted in Ref. [1] ; its presence was pointed out to me by J. Garriga [2]. In general, the boundary term is given by [3]

$$
S_{\text {boundary }}=-\frac{1}{8 \pi G} \partial_{\text {normal }}(\text { Volume of boundary }) \text {. }
$$

In our case, the volume of a 3 -sphere $\sigma=$ const is $2 \pi^{2} b^{3}(\sigma)$, and with the aid of (10) we obtain

$$
S_{E}=S_{\text {boundary }}=\left(\frac{3 \pi^{3}}{4 G}\right)^{1 / 2} C .
$$

Analytic continuation of the metric (2) to Lorentzian time, $\psi=\pi / 2+i t$, gives [1]

$$
d s^{2}=d \sigma^{2}+b^{2}(\sigma)\left(-d t^{2}+\cosh ^{2} t d \Omega_{2}^{2}\right) .
$$

It describes an asymptotically-flat spacetime with a singular hypersurface at $\sigma=\sigma_{*}$. The singularity has the form of a sphere expanding at a speed close to the speed of light. This can be seen by considering a sphere, $b(\sigma) \cosh t=R$, of a constant proper radius $R \gg\left(C / m_{p}\right)^{1 / 2}$. It is easily verified that this sphere is engulfed by the singularity in a finite proper time $\Delta \tau \approx R$ from the moment of nucleation $t=0$.

If the singular Euclidean solution described above is accepted as a legitimate instanton, we will have to conclude that flat space is unstable with respect to nucleation of singular bubbles. The nucleation probability is $\mathcal{P} \propto e^{-S_{E}}$ and is not exponentially suppressed for sufficiently small values of $C \lesssim m_{p}^{-1}$. If this picture were correct, most of the universe would have already been overrun by expanding singular bubbles. Since this picture is in glaring contradiction with observations, we have to conclude that singular instantons of the Hawking-Turok type cannot be used to describe quantum nucleation processes. In particular, they cannot describe nucleation of open universes. 
I am grateful to Jaume Garriga for very useful discussions during the course of this work.

Note added:

Hawking and Turok responded to the e-print version of this paper by pointing out that in their case the singularity never hits an observer in the open universe spacetime region, while in the case of my asymptotically flat instanton the singular bubble expands to engulf the whole space [4. It appears, however, that this observation is beside the point. The relevant question is: which instantons contribute to the Euclidean path integral? If singular instantons do contribute, then my instanton should be included as well, with all its unpleasant consequences. 


\section{REFERENCES}

[1] S.W. Hawking and N.G. Turok, "Open Inflation Without False Vacua", hep-th/9802030.

[2] J. Garriga, unpublished.

[3] G.W. Gibbons and S.W. Hawking, Phys. Rev. D15, 2752 (1977).

[4] N.G. Turok and S.W. Hawking, hep-th/9803156. 